\title{
Estabilización orbital de un robot móvil con ruedas tipo uniciclo: síntesis y validación experimental
}

\author{
Maugro Hernández-Ruiz ${ }^{1}$, Iliana Marlen Meza-Sánchez ${ }^{2}$ \\ 1 Tecnológico Nacional de México, Instituto Tecnológico de Ensenada, \\ Ensenada BC, México \\ ${ }^{2}$ Cátedra CONACYT, Instituto Tecnológico de Ensenada, \\ Ensenada BC, México,
}

mhernandez@ite.edu.mx, immezasa@conacyt.mx

\begin{abstract}
Resumen. En este trabajo se propone el desarrollo de un controlador por rediseño de Lyapunov para generar movimiento periódico en un robot móvil con ruedas (rmr) tipo uniciclo. Se utiliza la solución de un sistema dinámico de segundo orden que exhibe un ciclo límite para generar la órbita deseada; este sistema dinámico permite especificar el radio de la órbita y el sentido de giro mediante la configuración de dos parámetros. A partir de este análisis, se diseña un controlador basado en el modelo cinemático del robot móvil para asegurar su convergencia hacia el movimiento periódico deseado y se realiza un análisis de estabilidad para la dinámica en lazo cerrado. Se presentan resultados experimentales para demostrar la efectividad y robustez del esquema propuesto utilizando una plataforma de visión en tiempo real.
\end{abstract}

Palabras clave: Estabilización orbital, robot móvil tipo uniciclo, control por rediseño de Lyapunov.

\section{Orbital Stabilization for a Unicycle-Type Whee- led Mobile Robot: Synthesis and Experimental Validation}

\begin{abstract}
In this work, a Lyapunov-based synthesis and control design to induce periodic motion in a unicycle-type wheeled mobile robot (wmr) is under study. The solution of a second-order dynamical system is used as desired trajectory to achieve orbital stabilization. The selected dynamical reference system exhibits a limit cycle behavior which allows to move from one orbit to another by simply changing the parameters regarding radius of the orbit and direction of rotation. From this analysis, a kinematic tracking controller is designed for which closed-loop dynamics and stability analysis are provided to demonstrate control problem resolution based on error dynamics. Performance and robustness issues for the proposed framework are illustrated by experimental results using a real-time vision control system.
\end{abstract}


Keywords: Orbital estabilization, unicycle-type mobile robot, Lyapunov redesign control.

\section{Introducción}

La estabilización orbital en la Robótica es un problema de control que ha sido abordada en su mayoría para sistemas mecánicos y bípedos con la finalidad de generar trayectorias cíclicas parametrizadas entre eslabones así, como también en la generación de patrones de caminata $[1,2,3]$. Recientemente, sobre robots móviles con ruedas, se han desarrollado múltiples aplicaciones basados en este enfoque. Por ejemplo, en [4] se presenta el desarrollo de un controlador para navegación autónoma y evasión de obstáculos utilizando un método heurístico en línea combinado con un filtro extendido de Kalman (EKF) para generar elipses alrededor de los óbstaculos; un algoritmo de control óptimo para seguimiento y regulación para robots móviles diferenciales no holonómicos utilizando balizas de RF es descrito en [5]; el problema de estabilización por control adaptivo utilizando un sistema de visión es desarrollado en [6]. Problemas más complejos son abordados por ejemplo por [7], en donde se presenta una propuesta de solución para coordinación de múltiples robots en formación hacia un objetivo donde las trayectorias son generadas por un oscilador neuronal; la implementación de comportamientos cooperativos utilizando control descentralizado para seguimiento de trayectorias periódicas es propuesto en [8].

Como puede observarse, la estabilización orbital es un problema de control que permite el desarrollo de nuevas propuestas de solución para problemas cada vez más complejos. La teoría de control aborda este problema bajo dos esquemas principales; ya sea buscando soluciones periódicas dentro de la dinámica del sistema generadas por variación paramétrica como por ejemplo propone [9], o utilizando sistemas dinámicos de referencia que de manera intrínseca exhiban este comportamiento como se presenta en [10]. El trabajo que se presenta pertenece a esta última forma.

En esta propuesta, se desarrolla un controlador para resolver el problema de regulación con una trayectoria deseada que presenta velocidades de desplazamiento y movimiento angular constantes en estado estable. La generación de la trayectoria priódica está definida por un sistema dinámico de segundo orden que presenta una órbita estable de manera natural.

Tradicionalmente, la generación de movimiento periódico se define como la resolución de un problema de seguimiento para una trayectoria descrita por velocidades lineal y angular constantes (Véase por ejemplo [11]); sin embargo, en esta propuesta se toma ventaja de la dinámica de un sistema dinámico de referencia que converje a un órbita periódica, con sentido de giro y dirección configurables, para desarrollar un controlador que resuelve un problema de regulación para imponer estabilización orbital en un robot móvil con ruedas tipo uniciclo. En este caso, no es necesario garantizar la convergencia de los estados del robot 
ya que el objetivo de control está especificado con respecto al radio y dirección del movimiento deseado. La ventaja de establecer este planteamiento consiste en que el controlador no necesita compensar la velocidad de la trayectoria deseada y dado que el sistema dinámico de referencia converje a un ciclo límite, es suficiente con garantizar estabilidad asintótica para un problema de regulación.

El presente trabajo se encuentra organizado de la siguiente manera. En la Sección 2 se presenta la descripción del problema de control que se pretende resolver, se describe el modelo cinemático del robot móvil y el sistema dinámico de referencia. La síntesis de control basada en rediseño de Lyapunov y el análisis de estabilidad en lazo cerrado se presenta en la Sección 3. Los resultados experimentales utilizando una plataforma de visión con procesamiento en tiempo real para el sistema bajo estudio se muestra en Sección 3. Finalmente, resumimos las conclusiones y comentarios finales en la Sección 5.

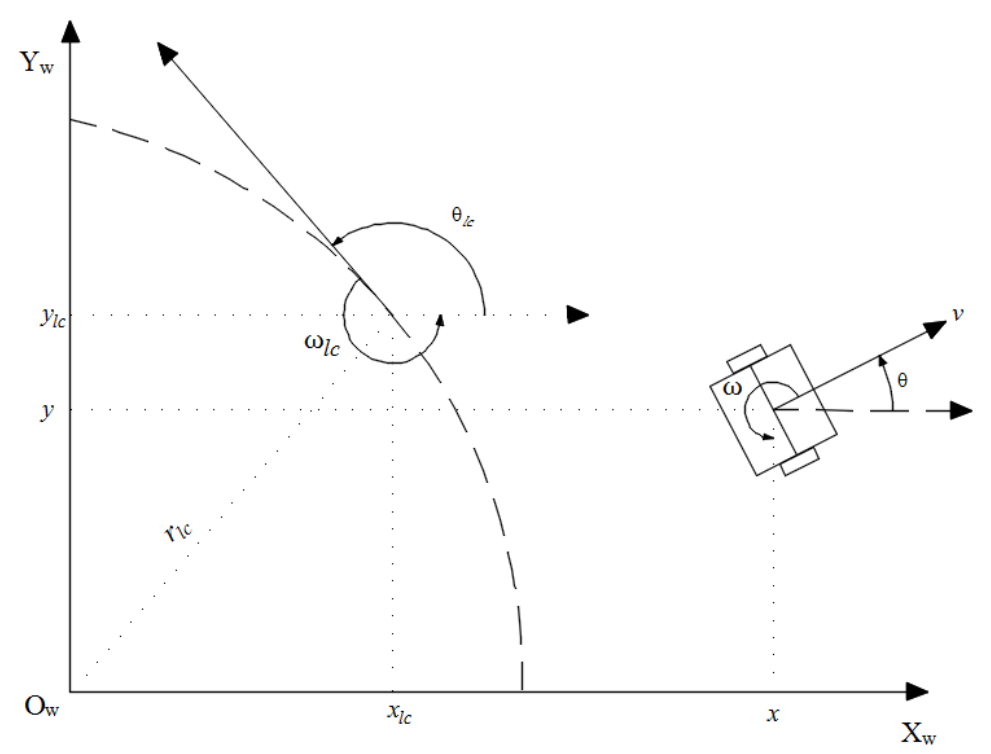

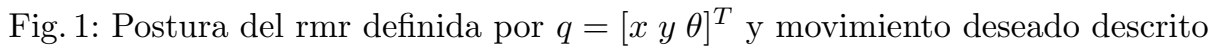
por $q_{l c}=\left[\begin{array}{lll}x_{l c} & y_{l c} & \theta_{l c}\end{array}\right]^{T}$ con respecto al marco fijo $X O Y_{\omega}$.

\section{Descripción del problema de control}

El objetivo de control es la generación de movimiento periódico en un robot móvil con ruedas tipo uniciclo por lo que se propone el uso de un sistema dinámico de segundo orden como modelo de referencia cuya dinámica converje a una trayectoria periódica alrededor del punto de equilibrio definido originalmente por 
el origen. A continuación, se presenta el modelo cinemático del rmr tipo uniciclo así como el modelo dinámico de referencia que ha sido seleccionado. En la Figura 1, se muestra el problema de control donde la trayectoria deseada y la postura del rmr están definidas por los vectores de estados $q_{l c}$ y $q$, respectivamente; ambas se encuentran descritas con respecto al marco fijo $\mathrm{XOY}_{w}$.

\subsection{Modelo cinemático}

Una de las principales características de los robots móviles con ruedas tipo uniciclo consiste en la restricción de movimiento en la cual sólo pueden desplazarse en dirección normal al eje de las ruedas. Esta característica propia de esta arquitectura puede expresarse matemáticamente como

$$
\dot{y} \cos \theta-\dot{x} \operatorname{sen} \theta=0,
$$

donde $\dot{x}, \dot{y}, \theta \in \mathbf{R}$ corresponden a las velocidades lineales en el eje $x$, en el eje $y, \mathrm{y}$ al ángulo de orientación, respectivamente. Considerando la restricción de movimiento descrita por (1), el modelo cinemático del robot móvil con respecto al marco fijo $\mathrm{XOY}_{w}$, está definido como

$$
\dot{q}=\left[\begin{array}{cc}
\cos \theta & 0 \\
\operatorname{sen} \theta & 0 \\
0 & 1
\end{array}\right] u
$$

donde las coordenadas generalizadas están descritas por el vector de estados $q=\left[\begin{array}{lll}x & y & \theta\end{array}\right]^{T}$, donde $x, y \in \mathbf{R}$ corresponden a las posiciones con respecto al plano $\mathrm{XY}_{w}$, y la orientación con respecto al eje $\mathrm{X}_{w}$ es descrito por el ángulo $\theta$; $u=[v \omega]^{T}$ define al vector de entradas de control denotado por las magnitudes de las velocidades lineal y angular, respectivamente.

\subsection{Modelo dinámico de referencia: ciclo límite}

El modelo seleccionado por exhibir un movimiento periódico configurable a través de dos parámetros para definir el radio de la órbita y el sentido de giro es el introducido en [10]; sin embargo, es importante señalar que existe una gran variedad de sistemas dinámicos clásicos y algunos propuestos en literatura que presentan este comportamiento y pueden ser utilizado bajo el esquema de esta propuesta.

El sistema de referencia seleccionado exhibe un ciclo límite y su dinámica está definida por

$$
\begin{aligned}
& \dot{x}_{l c}=x_{l c}-\delta y_{l c}-x_{l c}\left(x_{l c}^{2}+y_{l c}^{2}\right) \frac{1}{r_{l c}^{2}} \\
& \dot{y}_{l c}=\delta x_{l c}+y_{l c}-y_{l c}\left(x_{l c}^{2}+y_{l c}^{2}\right) \frac{1}{r_{l c}^{2}},
\end{aligned}
$$


donde $r_{l c} \in \mathbf{R}$ es una constante positiva que define el radio de la órbita $\mathrm{y}$ $\delta=\{+1,-1\}$ al sentido de rotación del movimiento. Redefínase este sistema de referencia $(3),(4)$, a un estructura equivalente dado por

$$
\dot{q}_{l c}=\left[\begin{array}{cc}
\cos \theta_{l c} & 0 \\
\operatorname{sen} \theta_{l c} & 0 \\
0 & 1
\end{array}\right]\left[\begin{array}{c}
v_{l c} \\
\omega_{l c}
\end{array}\right],
$$

donde $\dot{q}_{l c}=\left[\begin{array}{lll}\dot{x}_{l c} & \dot{y}_{l c} & \dot{\theta}_{l c}\end{array}\right]^{T} \in \mathbf{R}^{3}$, corresponde al vector de velocidades del movimiento en los ejes $x, y, X_{\omega}$, respectivamente; $v_{l c}, \omega_{l c} \in \mathbf{R}$ se redefinen como las velocidades lineal y angular de (3), (4).

La descripción de la reformulación definida en (5) puede ser obtenida mediante [9]

$$
v_{l c}=\sqrt{x_{l c}^{2}+y_{l c}^{2}}, \quad \omega_{l c}=\frac{\dot{x} \ddot{y}_{l c}-\dot{y}_{l c} \ddot{x}_{l c}}{\dot{x}_{l c}^{2}+\dot{y}_{l c}^{2}}, \theta_{l c}=\operatorname{atan} 2\left(\dot{y}_{l c}, \dot{x}_{l c}\right),
$$

donde $\theta_{l c}$ corresponde al ángulo de orientación del sistema dinámico considerandolo básicamente como si se tratara de un robot móvil de referencia; $\ddot{x}_{l c}, \ddot{y}_{l c}$ se refiere a las derivadas temporales de (3), (4), respectivamente.

\section{Síntesis de control y análisis de estabilidad}

Como se muestra en la Figura 1, el ángulo de orientación es periódico con un periodo definido por $T=2 \pi$. Además, considerando las propiedades del sistema de referencia definido por (3) y (4), el problema puede ser formulado como un control de posición a lo largo de la trayectoria deseada. Obsérvese que dado que el sistema de referencia posee un ciclo límite estable, esto implica que cualquier trayectoria, sin importar donde inicie, convergerá hacia una órbita de radio $r_{l c}$ en un sentido de rotación definido por $\delta=\{1,-1\}$.

En consecuencia, el objetivo de control está descrito como el diseño de una ley de control $(v, \omega)$, tal que el robot móvil converja a la órbita generada por (3), (4); esto es

$$
\lim _{t \rightarrow \infty}\left\|r_{l c}-r\right\|=0
$$

donde $k \in \mathbb{Z}$ y el error de la distancia en $x, y$ del robot móvil con respecto a la órbita están definidas por $r_{l c}=\sqrt{x_{l c}^{2}+y_{l c}^{2}}$ y $r=\sqrt{x^{2}+y^{2}}$.

Primero, defínase el vector de errores de estado $q_{e}=T(\theta)\left(q_{c}-q\right) \in \mathbf{R}^{3}$ donde $q=[x, y, \theta]^{T}, q_{c}=\left[x_{c}, y_{c}, \theta_{c}\right]^{T}$ corresponden a los vectores de postura del robot móvil y al de la trayectoria deseada, respectivamente. Por lo tanto, el error de posición está dado por

$$
q_{e}=T(\theta)\left(q_{c}-q\right)=\left[\begin{array}{ccc}
\cos \theta & \operatorname{sen} \theta & 0 \\
-\operatorname{sen} \theta & \cos \theta & 0 \\
0 & 0 & 1
\end{array}\right]\left[\begin{array}{l}
x_{e} \\
y_{e} \\
\theta_{e}
\end{array}\right]
$$


donde $x_{e}=\left(x_{c}-x\right), y_{e}=\left(y_{c}-y\right), \theta_{e}=\left(\theta_{c}-\theta\right)$. La dinámica del error puede ser obtenida a través de la derivada temporal de (8) como

$$
\dot{q}_{e}=\left[\begin{array}{cc}
\cos \theta_{e} & 0 \\
-\operatorname{sen} \theta_{e} & 0 \\
0 & 1
\end{array}\right]\left[\begin{array}{c}
v_{c} \\
\omega_{c}
\end{array}\right]+\left[\begin{array}{cc}
-1 & y_{e} \\
0 & -x_{e} \\
0 & -1
\end{array}\right]\left[\begin{array}{c}
v \\
\omega
\end{array}\right],
$$

o reformulada de manera equivalente,

$$
\begin{aligned}
& \dot{x}_{e}=v_{l c} \cos \theta_{e}+y_{e} \omega-v, \\
& \dot{y}_{e}=v_{l c} \operatorname{sen} \theta_{e}-\omega x_{e} \\
& \dot{\theta}_{e}=\omega_{e}
\end{aligned}
$$

donde $\omega_{e}=\omega_{l c}-\omega$.

Para garantizar el cumplimiento del objetivo de control imponiendo la convergencia de la dinámica del error (8) a la variedad (7), se propone una ley de control dada por

$$
\begin{aligned}
v & =v_{l c} \cos \theta_{e}+K_{x} x_{e} \\
\omega & =\omega_{l c}+K_{\omega} v_{l c} y_{e}+K_{\omega} \operatorname{sen} \theta_{e}\left(1+K_{y} y_{e}^{2}\right)
\end{aligned}
$$

donde $K_{x}, K_{y}, K_{\omega}>0$.

Es importante señalar que la propuesta de control resuelve el problema de regulación de postura del robot móvil con respecto al sistema de referencia que exhibe un ciclo límite estable; i.e. el objetivo es garantizar estabilidad con respecto a (7).

Teorema 1. Sea el sistema libre de perturbaciones (2) restringido por (1) y con dinámica de error definida por (8), donde se aplica la ley de control descrita por (12), (13) y está sujeto a una trayectoria definida como una sucesión de posiciones con respecto a la solución del sistema dinámico de segundo orden (5). Entonces, el sistema en lazo cerrado dado por (2), (12), (13) es globalmente asintóticamente estable con $K_{x}, K_{y}, K_{\omega}>0$.

Demostración. Propóngase la siguiente función candidata

$$
V=\frac{1}{2}\left(x_{e}^{2}+y_{e}^{2}\right)+K_{\omega}^{-1}\left(1-\cos \theta_{e}\right)
$$

donde $K_{\omega}>0$ es una constante positiva. Entonces, la derivada temporal de (14) está dada por

$$
\dot{V}=x_{e} \dot{x}_{e}+y_{e} \dot{y}_{e}+K_{\omega}^{-1} \operatorname{sen} \theta_{e} \dot{\theta}_{e} .
$$

Tomando en cuenta los errores en la dinámica (9), (10), (11), y sustituyendo (12), (13) en (15) tenemos

$$
\dot{V}=-K_{x} x_{e}^{2}-K_{\omega}^{-1} \operatorname{sen}^{2} \theta_{e}\left(1+K_{y} y_{e}^{2}\right) \leq 0
$$

$\operatorname{con} K_{x}, K_{y}, K_{\omega}>0 \in \mathbf{R}$. 


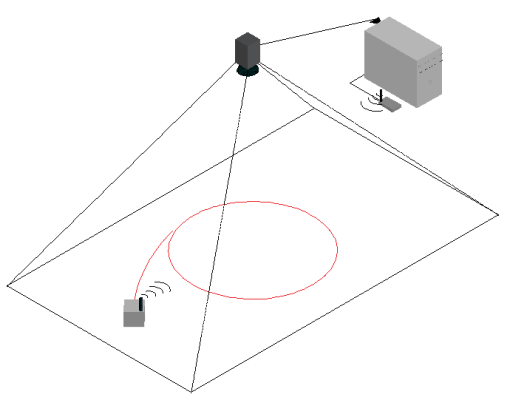

(a)

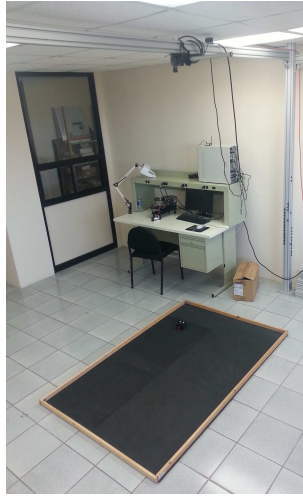

(b)

Fig. 2: Configuración experimental utilizando una plataforma de visión con procesamiento en tiempo real: (a) esquema, b) sistema físico de laboratorio.

Inspirado en la línea de razonamiento de [9], podemos establecer que dada (16), la función candidata de Lyapunov propuesta $V(t)$ definida en (14) es decreciente y por lo tanto, posee un límite superior conforme $t \rightarrow \infty$; i.e. (14) es una función $\mathcal{L}_{\infty}$. Esta propiedad permite concluir entonces que el vector de error de estados $\left[\begin{array}{lll}x_{e} & y_{e} & \theta_{e}\end{array}\right]^{T}$, sus derivadas temporales $\left[\begin{array}{lll}\dot{x}_{e} & \dot{y}_{e} & \dot{\theta}_{e}\end{array}\right]^{T}$, así como tambiíen la ley de control (12),(13) son funciones $\mathcal{L}_{\infty}$ dado que $K_{x}, K_{y}, K_{\omega}$ son constantes y $v_{l c}, \omega_{l c}$ del sistema de referencia también son funciones acotadas. La estabilidad global asintótica para $x_{e}$ y $\theta_{e}$ puede ser rápidamente concluida mediante el cálculo de la integral de (16) para establecer que $x_{e}$ y $\theta_{e}$ son funciones $\mathcal{L}_{2}$, y aplicar la versión extendida del Lema de Barbalat (Véase Apéndice A).

Con el resultado anterior, dado que $\lim _{t \rightarrow \infty} \theta_{e}=0$ y la igualdad (11) se cumple, la dinámica del error para la velocidad angular $\omega_{e}$ tambien converge a cero; por lo tanto, sustituyendo (13) en (11) se obtiene que $\lim _{t \rightarrow \infty} K_{\omega} v_{l c} y_{e} \rightarrow 0$. En consecuencia, se concluye también que $\lim _{t \rightarrow \infty} y_{e}=0$ debido a que $v_{l c}$ es uniformemente continua y diferente de cero, y $K_{\omega}$ es una constante positiva. Resumiendo ambos resultados, el sistema en lazo cerrado (2), (12), (13) es globalmente asintóticamente estable y la validez del Teorema 1 queda demostrada.

Remark 1. Considerando que el origen del sistema dinámico de segundo orden utilizado como referencia es un punto de equilibrio inestable, el robot móvil no puede ser inicializado en este punto. En la práctica, pequeñas perturbaciones y errores de precisión numérica en las mediciones permiten evitar esta situación; además es posible agregar de manera artificial una pequeña desviación a la medición de postura. 

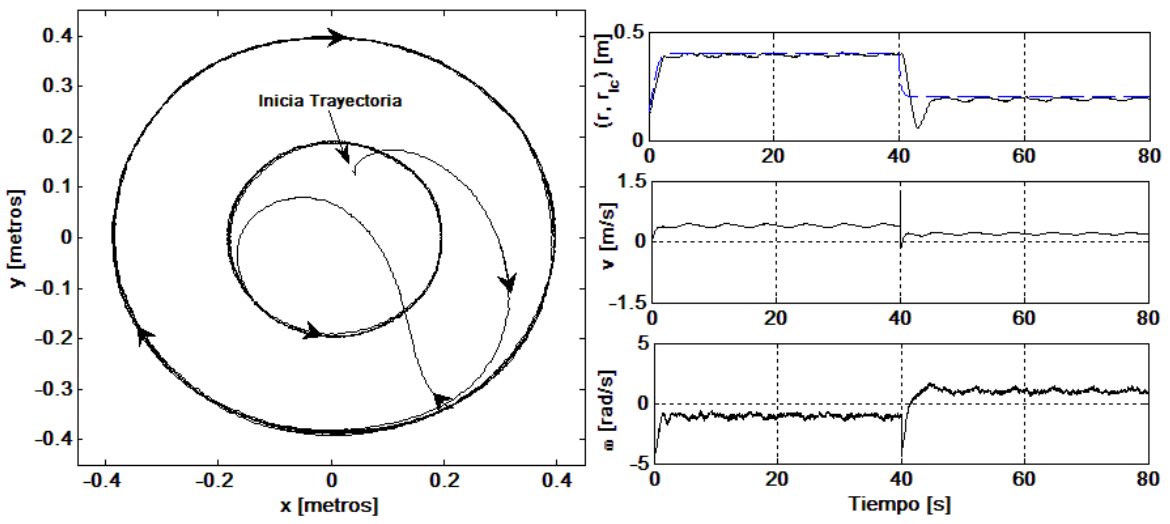

Fig. 3: Resultado experimental para el caso sin perturbaciones con condiciones iniciales $q(0)=\left[\begin{array}{lll}0,0394 & 0,1316 & 2,338\end{array}\right]^{T}$. En $\left(r, r_{l c}\right)$, la línea sólida corresponde al radio $r[\mathrm{~m}]$ del movimiento de rmr y la punteada al radio $r_{l c}=\{0.25,0.4\}[\mathrm{m}]$ del sistema de referencia (3),(4) con $\delta=\{-1,1\}$.

\section{Resultados experimentales}

Se realizaron experimentos utilizando una plataforma de visión en tiempo real para validar la efectividad del diseño de control propuesto. Esta plataforma se encuentra en el Laboratorio de Robótica Avanzada del Instituto Tecnológico de Ensenada basada en el sistema propuesto en [12] y se muestra en la Figura 2. Este sistema utiliza comunicación inalámbrica de RF para cerrar el lazo de control mediante un transmisor conectado al puerto paralelo de la $\mathrm{PC}$ y el tiempo de muestreo está definido como $t_{m}=0.015[\mathrm{~s}]$.

Los experimentos realizados consideran dos casos: a) el caso no perturbado en donde el robot móvil se mueve libremente de una órbita hacia otra, y b) el caso perturbado en donde manualmente se altera la trayectoria del robot móvil sacandolo de la órbita deseada. La trayectoria de referencia es generada mediante una integración numérica con un tiempo de muestreo $t_{m}=0.015$ [s] en la plataforma de visión en tiempo real para obtener la solución del sistema dinámico de referencia.

Las condiciones iniciales para los experimentos sin perturbaciones y el perturbado son $q_{1}(0)=\left[\begin{array}{llll}0,0394 & 0,1316 & 2,338\end{array}\right]^{T}, q_{2}(0)=\left[\begin{array}{lll}-0,6101 & 0,1910 \quad-\end{array}\right.$ $0,3591]^{T}$, respectivamente. Las mediciones para $x$ y $y$ son en metros, y en radianes para el ángulo de orientación $\theta$. Las velocidades iniciales fueron definidas como $q_{i}=\left[\begin{array}{lll}0 & 0 & 0\end{array}\right]^{T}, i=1,2$. El subíndice 1,2 se utiliza para el caso sin perturbaciones y el caso perturbado, respectivamente. Los valores de los parámetros del sistema dinámico de referencia son $r_{l c}=0.4[\mathrm{~m}]$ y $\delta=-1$ para los primeros $40[\mathrm{~s}]$, para posteriormente cambiar la órbita a un radio de $r_{l c}=0.25$ [m] e invertir el sentido de giro con $\delta=1$. Las ganancias seleccionadas para el 

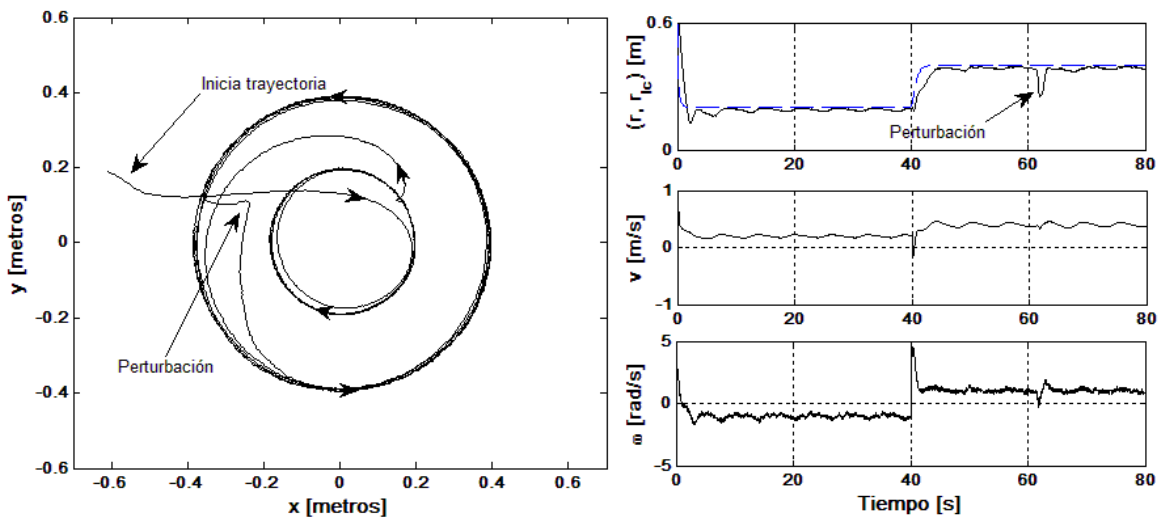

Fig. 4: Resultado experimental para el caso perturbado en $t \approx 63[\mathrm{~s}]$ con condiciones iniciales $q(0)=\left[\begin{array}{lll}-0,6101 & 0,1910 & -0,3591\end{array}\right]^{T}$. En $\left(r, r_{l c}\right)$, la línea sólida corresponde al radio $r[\mathrm{~m}]$ del movimiento de $\mathrm{rmr}$ y la punteada al radio deseado $r_{l c}=\{0.25,0.4\}[\mathrm{m}]$ del sistema de referencia $(3),(4) \operatorname{con} \delta=\{-1,1\}$.

controlador (12), (13) fueron $K_{x}=0,4, K_{y}=1$, y $K_{\omega}=3$.

El resultado experimental para el caso no perturbado se muestra en la Figura 3 donde el controlador propuesto cumple con el objetivo de control estabilizando asintóticamente el movimiento periódico sobre el robot móvil. Además, en el inicio de su trayectoria presenta un desplazamiento en reversa para incorporarse a la órbita deseada. La gráfica $\left(r, r_{l c}\right)$ muestra la comparación entre el radio deseado y el radio generado por el movimiento del rmr; $v$ y $\omega$ muestran las velocidades lineal y angular del robot. El resultado del experimento para el caso perturbado se muestra en la Figura 4; para este experimento, se desplaza manualmente al robot fuera de la órbita aproximadamente en $t=63[\mathrm{~s}]$ y el controlador logra que este recupere el movimiento periódico deseado. De ambas Figuras, podemos observar también que se logra la estabilización orbital sin importar si la postura inicial de robot móvil se encuentra fuera o dentro de la órbita deseada.

\section{Conclusión y comentarios finales}

Se ha presentado el diseño y la validación experimental de un controlador para regulación basado en rediseño de Lyapunov para inducir estabilización orbital en un robot móvil con ruedas tipo uniciclo. Además, se ha demostrado estabilidad asintótica del sistema en lazo cerrado. Los resultados experimentales utilizando una plataforma de visión con procesamiento en tiempo real ha demostrado su eficiencia al lograr el objetivo de control para un movimiento deseado generado por un sistema dinámico de referencia de segundo orden a pesar de que la solución de este último ha sido integrada numéricamente. El trabajo futuro 
pretende extender la estrategia de control propuesta para resolver problemas más complejos como control de formación y navegación autónoma mediante la generación de campos potenciales definidos por ciclos límites alrededor de los obstáculos.

Agradecimientos. Los autores desean agradecer al CONACYT Proyecto Cátedras 2459 denominado "Redes de Robots Móviles Colaborativos", al Tecnológico Nacional de México, al Instituto Tecnológico de Ensenada y a PRODEP.

\section{A. Lema de Barbalat y versión extendida}

El lema de Barbalat es un resultado teórico bien conocido utilizado para analizar estabilidad en sistemas no lineales. Su forma original y su versión extendida se definen a continuación $[9,13]$.

Lemma 1. Sea $f: \mathbf{R} \rightarrow \mathbf{R}$ una función uniformemente continua en $[0, \infty)$.

Supóngase que $\operatorname{lím}_{t \rightarrow \infty} \int_{0}^{t} f(\tau) d \tau$ existe y es finito. Entonces, $f(t) \rightarrow 0$ conforme $t \rightarrow \infty$.

Lemma 2. Sea $f, \dot{f} \in \mathcal{L}_{\infty}$ y $f \in \mathcal{L}_{p}$ para alguna $p=[1, \infty)$ entonces $f(t) \rightarrow 0$ conforme $t \rightarrow \infty$.

\section{Referencias}

1. Meza-Sánchez I.M., Aguilar L.T., Shiriaev A., Freidovich L., Orlov Y.: Periodic motion planning and nonlinear H-infinity tracking control of a 3-DOF underactuated helicopter. Int Journal of Syst Science 42(5), 829-838 (2011)

2. Garofalo G., Ott C., Albu-Schaffer A.: Orbital stabilization of mechanical systems through semidefinite Lyapunov functions. In: American Control Conference (ACC), pp. 5715-5721, Washington, DC, USA (2013)

3. Gamus B., Or Y.: Dynamic Bipedal Walking under Stick-Slip Transitions. SIAM (Society for Industrial and Applied Mathematics) J. Applied Dynamical Systems, 14(2), pp. 609--642 (2015)

4. Vilca J., Adouane L., Mezouar Y.: Reactive Navigation of a Mobile Robot Using Elliptic Trajectories and Effective Online Obstacle Detection. Gyroscopy and Navigation, 4(1), pp. 14-25 (2013)

5. Miah M.S., Gueaieb W.: RFID-Based Mobile Robot Trajectory Tracking and Point Stabilization Through On-line Neighboring Optimal Control. J Intell Robot Syst, 78, pp. 377--399 (2015)

6. Yang F., Wang C.: Adaptive stabilization for nonholonomic mobile robots with uncertain dynamics and unknown visual parameters. Transactions of the Institute of Measurement and Control, 37(2), pp. 282-288 (2015)

7. Sajjad M., Youngjin C: Neural oscillator-based multi-robot coordination algorithm to catch-Observe-Protect a target. In: IEEE Int Conf on Mechatronics and Automation (ICMA), pp. 1418-1423, Beijing, China (2015) 
Estabilización orbital de un robot móvil con ruedas tipo uniciclo: síntesis y validación experimental

8. Sabattini L., Secchi C., Cocetti M., Levratti A., Fantuzzi C.: Implementation of Coordinated Complex Dynamic Behaviors in Multirobot Systems. IEEE Trans on Robotics, 31(4), 1018-1032 (2015)

9. Blazic, S.: On Periodic Control Laws for Mobile Robots. IEEE Trans on Industrial Electronics, 61(7), 3660-3670 (2014)

10. Hara N., Kokame H., Konishi K.: Circular Periodic Motion Generation for Mobile Robots Using Limit Cycle Systems. In: American Control Conference (ACC), pp. 4271-4276, Baltimore, USA (2010)

11. Wang Y., Miao Z., Zhong H., Pan Q.: Simultaneous Stabilization and Tracking of Nonholonomic Mobile Robots: A Lyapunov-Based Approach. IEEE Trans on Control Systems Technology, 23(4), pp. 1440-1450 (2015)

12. Bugarin E., Kelly R.: RTSVC: Real-time system for visual control of robots. Int. J. Imaging Syst. Technol., 18, 251-256 (2008)

13. Khalil H. K.: Nonlinear Systems. Second edition, Prentice-Hall (1996) 